\title{
NONLINEAR NONEQUILIBRIUM NONQUANTUM NONCHAOTIC STATISTICAL MECHANICS OF NEOCORTICAL INTERACTIONS
}

\author{
Lester Ingber \\ Lester Ingber Research \\ P.O. Box 857 \\ McLean, VA 22101 \\ ingber@alumni.caltech.edu
}

\begin{abstract}
The work in progress reported by Wright \& Liley shows great promise, primarily because of their experimental and simulation paradigms. However, their tentative conclusion that macroscopic neocortex may be considered (approximately) a linear nearequilibrium system is premature and does not correspond to tentative conclusions drawn from other studies of neocortex.
\end{abstract}

KEYWORDS: EEG; nonlinear, statistical;

\section{IMPORTANCE OF APPROACH}

At this time, there exists an interdisciplinary multidimensional gradation on published studies of neocortex, with one primary dimension of mathematical physics represented by two extremes. At one extreme, there is much scientifically unsupported talk of chaos and quantum physics being responsible for many important macroscopic neocortical processes (involving many thousands to millions of neurons) (Wilczek, 1994). At another extreme, many non-mathematically trained neuroscientists uncritically lump all neocortical mathematical theory into one file, and consider only statistical averages of citations for opinions on the quality of that research (Nunez, 1995). In this context, it is important to appreciate that Wright and Liley (W\&L) report on their scientifically sound studies on macroscopic neocortical function, based on simulation and a blend of sound theory and reproducible experiments.

However, their pioneering work, given the absence of much knowledge of neocortex at this time, is open to criticism, especially with respect to their present inferences and conclusions. Their conclusion that EEG data exhibit linear near-equilibrium dynamics may very well be true, but only in the sense of focusing only on one local minima, possibly with individual-specific and physiological-state dependent 
parameters, embedded in a more relevant global context. It certainly would be more extreme, and probably quite incorrect, to conclude from W\&L's inference that macroscopic neocortical phenomena in general can be treated as linear near-equilibrium systems.

\section{NO CHAOS}

As discussed by W\&L, more evidence is being collected on the irrelevance of chaos to macroscopic neocortical interactions; this is similar to serious work undertaken in several fields, where the impulse to identify "chaos" in a complex system has often proved premature (Rapp et al, 1993). It is not supported by the facts, tentative as they are, because of sparse data. Similar caution should be exercised regarding neocortical interactions.

Many such studies demonstrating chaos are quite model dependent; hence it is fair to present only the models as exhibiting chaos, not necessarily the actual physical system. There are, however, reports of model-independent experimental observations of distinctive stimulus-dependent parameters with extreme sensitivity to initial conditions.

It has been widely noted that measures such as the correlation dimension of data are difficult to calculate (Abarbanel et al, 1993). Perhaps the correlation dimension is often not even a well-founded concept, for example, since the EEG of event-related potentials is probably nonstationary and highly context- and subject-dependent. Its calculation, even when supplemented with finer statistical tests and noise reduction techniques, may prove fruitful, but probably only as a sensitivity index relative to shifting contexts and complementary to other models of EEG data.

Early in the development of a model of the statistical mechanics of neocortical interactions (SMNI) (Ingber, 1981; Ingber, 1982), it was noted that remarkable sensitivity of macroscopic phenomena would be required to render any chaos that might exist at microscopic scales relevant at columnar or macroscopic scales (Ingber, 1983).

In a recent study using a highly accurate algorithm to evolve multivariate probability distributions, chaos persisted in the presence of moderate noise in a Duffing analogue of EEG systems (Ingber, Srinivasan \& Nunez, 1995). It was tentatively concluded (more CPU time is needed for longer detailed runs) that adding moderate noise to models of neocortical systems that might exhibit (highly modeldependent) chaos washes out traces of chaos. 


\section{NO QUANTUM MECHANICS}

Quite often, quantum mechanics is invoked to "explain" the linearity of macroscopic information processing under some set of hypothesized circumstances, for example, preparatory to invoking the quantum mechanical principle of superposition. However, there does not seem to be any reasonable role for specific quantum processes explaining any macroscopic neocortical processes (certainly at the levels of phenomena giving rise to scalp EEG, consciousness, etc.) (Wilczek, 1994).

One often get queries concerning associations between quantum mechanics and SMNI, mostly because of the similarities in their mathematical structures. It must be made clear that SMNI and quantum theory are only related to the extent that some modern methods of classical nonlinear nonequilibrium statistical mechanics have developed a modern calculus in the late 1970s (Graham, 1978; Langouche et al, 1982), that turns out to have been used likewise for some phenomena (too small to measure) in quantum gravitational physics (DeWitt, 1957). SMNI also has relevance to some measurable phenomena in nuclear physics (Ingber, 1984a). There is absolutely no other physical relationship between SMNI and quantum mechanics. For example, there are some subtle quantum mechanical processes in information processing that are specific to quantum mechanics that have no relation to any possible classical information processing (Bennett, 1995).

In this context, it is noteworthy that the modern calculus, used for many other systems as well as for SMNI, does not even need the usual ensemble-averaging statistical mechanical arguments for its development, for example, as is implicit in Langevin-type differential stochastic rate equations in which "noise" reflects on finer degrees of freedom than the order parameters. Rather, the mathematically equivalent Fokker-Planck ("Schrödinger-type") and Lagrangian path-integral representations, which can be derived by ensemble averages of such noise, can be directly derived by nonlinear nonequilibrium thermodynamic arguments (Ruppeiner, 1995).

\section{NONLINEAR NONEQUILIBRIUM}

It is hard to understand why a claim is made for linearity across the range of phenomena measured by EEG data, if even those data have the requisite resolving power to tell us much about neocortical function. This last condition is not trivially established, and is of course a subject of intense research. 
For example, the existence of multiple attractors must certainly be taken as evidence for nonlinearity, by definition. Short-term memory (STM) certainly exhibits multiple states (Miller, 1956; Zhang \& Simon, 1985), and it seems reasonable to conclude that this is due to nonlinearity in neocortical processing (Ingber, 1984b; Ingber, 1985; Ingber, 1994; Ingber, 1995a; Ingber \& Nunez, 1995; Ingber, 1995b; Ingber, 1996b).

It is possible, but unlikely, that multiple states of STM are due to a single attractor, driven to extreme nonequilibrium within tenths of seconds (to access multiple memories within these epochs, as observed) to new contexts (thereby at least contradicting any claims of near-equilibrium!). For example, there is strong evidence for the existence of mechanisms that establish at least quasi-stationarity of multiple attractors (Lisman \& Idiart, 1995; Ingber, 1995b).

The reference in section 3 of $\mathrm{W} \& \mathrm{~L}$ to the confluence of global and local interactions (Ingber $\&$ Nunez, 1990) in fact argues against linearity of EEG-measured mechanisms. A more specific study, directly related to that reference, demonstrated that EEG could be fit quite well by SMNI, using only values of synaptic and neuronal parameters lying within experimentally determined ranges (Ingber, 1991). This required the use of a sophisticated global optimization technique, Adaptive Simulated Annealing (ASA) (Ingber, 1993), previously called Very Fast Simulated Reannealing (VFSR) (Ingber, 1989) was used to fit the global optimum over several attractors, specific to each subject's P300 data, within the group of 49 subjects. It was certainly clear from that study that there is a great deal of nonequilibrium dynamics inherent in neocortex; for example, these attractors would radically shift if one attempted the same study using other epochs of EEG.

A good analogue exists to demonstrate just how EEG might have to look to be linear. The same mathematical development used for SMNI has been applied to financial markets, including specific trading scenarios (Ingber, 1984c; Ingber, 1990; Ingber, 1995d; Ingber, 1996c). The financial data are well-known to be quite nonlinear and nonstationary (in fact, they are quite hard to distinguish visually from some EEG data!). Global optimization, such as using ASA (Ingber, 1993), is required to fit these bivariate nonlinear stochastic models to data. However, if decisions are to made on recognizing patterns in the data, that is, for the purposes of trading, then trading rules based on a kind of moving average of "canonical momenta" indicators (derived from the Lagrangian model fit to a separate independent training set of data) can indeed take advantage of "inefficiencies" in the market, where inefficiencies here imply 
nonlinear nonequilibrium states of the system.

A current project uses similar constructs for canonical momentum indicators derived for EEG from the SMNI Lagrangian as previously fit to EEG data (Ingber, 1991), to correlate physiological or behavioral states in human subjects (Ingber, 1995c; Ingber, 1996a). It is recognized, however, that this will require "customization" for each subject. Similar to the recursive optimization performed for the above finance calculations, there is an inner shell of optimization on the Lagrangian model to data, and an outer shell of optimization on the rules for pattern recognition-in this case parameterized rules used by clinicians.

The point here is that the use of moving averages might seem to imply that the system is being viewed as linear and near-equilibrium. However, this would be quite myopic, looking only within a short window of data, instead of seeing or appreciating the full context of the system. This myopic view, if implemented in trading, without the possibility of adjusting in a timely fashion to indications that the system is quite nonlinear and nonequilibrium, would quickly lead to financial ruin. I believe it is fair to state that a similar view of EEG would quickly lead to incorrect diagnoses of many physiological and behavioral states across many patients and subjects.

\section{QUESTIONS}

Some questions can be raised specific to $\mathrm{W} \& \mathrm{~L}$, with a view to constructive improvement of their methodology. They outline some of their basic assumptions in section 4.1. Are all the parameters and variables in their assumed stochastic second-order differential equations tightly bound to specific neuronal entities, within experimentally established ranges? This is not clear for all parameters, notwithstanding their presentation of Table 1. Otherwise, some other system may be being fit.

(Piece-wise) Linear models of data can usually be expected to extract linear systematics. It would be more useful to develop some realistic nonlinear model, and then to see whether it could exhibit a specific range that strongly exhibits linearity.

The use of "white noise" for W\&L's simulations suffers serious defects. It has long been recognized that many complex chemical and biological systems have complex noise structures (van Kampen, 1976), typically arising from stochastics and statistics of variables and parameters arising in the "deterministic" structures. This certainly was true in SMNI development. 
In this context, $\mathrm{W} \& \mathrm{~L}$ have taken their parameters to be time-independent as well as noise independent, to facilitate implementation of their model. In many complex systems, one can often use time-independent parameters if one takes into account that nonlinear nonequilibrium structures are required to model temporal evolution. Attempts to approximate these systems with quasilinear models, (e.g., ARMA-type models) requires including many more higher-order structures to model this timedependence (Kishida, 1982; Kishida, 1984).

The point here is that for many systems, only after a truly nonlinear study is undertaken (one with a full nonlinear structure in the noise) does it make sense to assume that one has found a local minimum ("the needle in the haystack"), and similarly for ranges of constant noise.

Most serious is the fact that a crucial and influential parameter in the W\&L model, the synaptic gain of set of questionable origin, has no specific neocortical origin. Anything might be expected, and W\&L seem to find quite a bit of activity by tweaking this parameter.

It is important to note that W\&L have undertaken further studies using the Genesis simulation, which can generate a good deal of of microscopic realistic neocortical modeling. It remains to be seen whether they can carry out this program of basing conclusions about macroscopic neocortex on sampling such microscopic simulations. Not only must columnar dynamics be properly modeled, with a thousand minicolumns per macrocolumn, but global effects from long-range firings are likewise critical in neocortical EEG studies. For example, the macroscopic SMNI model, derived from a progression of development at neuronal and columnar scales, requires specific optimization, path-integral, and parallel algorithms to treat its minicolumnar network model (Ingber, 1992). This model has taken advantage of the particular class of nonlinear structures many complex systems develop at mesoscales (GaussianMarkovian only in short epochs) and it remains to be seen how other complex models can be accurately calculated.

Like other complex systems (even "simple" fluids), a system as complex as neocortex is best treated as an overlap of quite different models at different spatial-temporal resolutions. I think W\&L basically agree with this. The scientific challenges are to establish strong theoretical and experimental bases for overlaps of these models at different scales, and to articulate and understand the precise nature of emergent variables, parameters, and structures across these scales. It is dubious that much will be learned by attempting a "grand simulation" of such systems, especially since such simulations and models 
typically require approximations too harsh for specific calculations and empirical tests.

W\&L are presumably undertaking the challenge of modeling macroscopic neocortex, respecting the importance of multiple scales of neocortical processing, and I assume the model they are developing presumably will be useful for future EEG data that will have better spatial resolution than they do today. These future resolutions are likely to lead to better correlations of EEG with real and individual human physiological and behavioral states, but then these models will have an even greater need to address nonlinear and nonequilibrium processes of neocortical interactions.

\section{REFERENCES}

Abarbanel, H.D.I., Brown, R., Sidorowich, J.J. \& Tsimring, L.Sh. (1993) The analysis of observed chaotic data in physical systems. Rev. Mod. Phys. 65:1331-1392.

Bennett, C.H. (1995) Quantum information and computation. Physics Today 48:24-30.

DeWitt, B.S. (1957) Dynamical theory in curved spaces. I. A review of the classical and quantum action principles. Rev. Mod. Phys. 29:377-397.

Graham, R. (1978) Path-integral methods on nonequilibrium thermodynamics and statistics, In: Stochastic Processes in Nonequilibrium Systems, ed. L. Garrido, P. Seglar and P.J. Shepherd. Springer, 82-138.

Ingber, L. (1981) Towards a unified brain theory. J. Social Biol. Struct. 4:211-224.

Ingber, L. (1982) Statistical mechanics of neocortical interactions. I. Basic formulation. Physica D 5:83-107.

Ingber, L. (1983) Statistical mechanics of neocortical interactions. Dynamics of synaptic modification. Phys. Rev. A 28:395-416.

Ingber, L. (1984a) Path-integral Riemannian contributions to nuclear Schrödinger equation. Phys. Rev. D 29:1171-1174.

Ingber, L. (1984b) Statistical mechanics of neocortical interactions. Derivation of short-term-memory capacity. Phys. Rev. A 29:3346-3358.

Ingber, L. (1984c) Statistical mechanics of nonlinear nonequilibrium financial markets. Math. Modelling 5:343-361. 
Ingber, L. (1985) Statistical mechanics of neocortical interactions: Stability and duration of the $7 \pm 2$ rule of short-term-memory capacity. Phys. Rev. A 31:1183-1186.

Ingber, L. (1989) Very fast simulated re-annealing. Mathl. Comput. Modelling 12:967-973.

Ingber, L. (1990) Statistical mechanical aids to calculating term structure models. Phys. Rev. A 42:7057-7064.

Ingber, L. (1991) Statistical mechanics of neocortical interactions: A scaling paradigm applied to electroencephalography. Phys. Rev. A 44:4017-4060.

Ingber, L. (1992) Generic mesoscopic neural networks based on statistical mechanics of neocortical interactions. Phys. Rev. A 45:R2183-R2186.

Ingber, L. (1993) Adaptive Simulated Annealing (ASA). [ftp.alumni.caltech.edu: /pub/ingber/ASA-shar, ASA-shar.Z, ASA.tar.Z, ASA.tar.gz, ASA.zip]. Lester Ingber Research.

Ingber, L. (1994) Statistical mechanics of neocortical interactions: Path-integral evolution of short-term memory. Phys. Rev. E 49:4652-4664.

Ingber, L. (1995a) Statistical mechanics of multiple scales of neocortical interactions, In: Neocortical Dynamics and Human EEG Rhythms, ed. P.L. Nunez. Oxford University Press, 628-681.

Ingber, L. (1995b) Statistical mechanics of neocortical interactions: Constraints on $40 \mathrm{~Hz}$ models of short-term memory. Phys. Rev. E 52:4561-4563.

Ingber, L. (1995c) Statistical mechanics of neocortical interactions (SMNI). SMNI Lecture Plates. Lester Ingber Research.

Ingber, L. (1995d) Statistical mechanics of nonlinear nonequilibrium financial markets: Applications to optimized trading. Mathl. Computer Modelling (to be published).

Ingber, L. (1996a) Adaptive simulated annealing of canonical momenta indicators of financial markets. (submitted) .

Ingber, L. (1996b) Statistical mechanics of neocortical interactions: Multiple scales of EEG. Electroencephal. clin. Neurophysiol. (to be published).

Ingber, L. (1996c) Trading markets with canonical momenta and adaptive simulated annealing. (submitted) . 
Ingber, L. \& Nunez, P.L. (1990) Multiple scales of statistical physics of neocortex: Application to electroencephalography. Mathl. Comput. Modelling 13:83-95.

Ingber, L. \& Nunez, P.L. (1995) Statistical mechanics of neocortical interactions: High resolution pathintegral calculation of short-term memory. Phys. Rev. E 51:5074-5083.

Ingber, L., Srinivasan, R. \& Nunez, P.L. (1995) Path-integral evolution of chaos embedded in noise: Duffing neocortical analog. Mathl. Computer Modelling (to be published).

Kishida, K. (1982) Physical Langevin model and the time-series model in systems far from equilibrium. Phys. Rev. A 25:496-507.

Kishida, K. (1984) Equivalent random force and time-series model in systems far from equilibrium. $J$. Math. Phys. 25:1308-1313.

Langouche, F., Roekaerts, D. \& Tirapegui, E. (1982) Functional Integration and Semiclassical Expansions. Reidel.

Lisman, J.E. \& Idiart, M.A.P. (1995) Storage of $7 \pm 2$ short-term memories in oscillatory subcycles. Science 267:1512-1515.

Miller, G.A. (1956) The magical number seven, plus or minus two. Psychol. Rev. 63:81-97.

Nunez, P.L. (1995) Neocortical Dynamics and Human EEG Rhythms. Oxford University Press.

Rapp, P.E., Albano, A.M., Schmah, T.I. \& Farwell, L.A. (1993) Filtered noise can mimic low dimensional chaotic attractors. Phys. Rev. E 47:2289-2297.

Ruppeiner, G. (1995) Riemannian geometry in thermodynamic fluctuation theory. Rev. Mod. Phys. 67:605-659.

van Kampen, N.G. (1976) Fluctuations in closed and open non-linear systems, In: Statistical Physics, ed. L. Pál and P. Szépfalusy. North-Holland, 29-48.

Wilczek, F. (1994) A call for a new physics. Science 266:1737-1738.

Zhang, G. \& Simon, H.A. (1985) STM capacity for Chinese words and idioms: Chunking and acoustical loop hypotheses. Memory \& Cognition 13:193-201. 